\title{
To Be Is To Become. Fractal Neurodynamics of the Body-Brain Control System
}

\author{
Franca Tecchio ${ }^{1 *}$, Massimo Bertoli ${ }^{1,2}$, Eugenia Gianni ${ }^{1,3}$, Teresa L'Abbate ${ }^{1}$, Luca Paulon ${ }^{1}$ \\ and Filippo Zappasodi ${ }^{2}$ \\ ${ }^{1}$ Laboratory of Electrophysiology for Translational NeuroScience, Institute of Cognitive Sciences and Technologies-Consiglio \\ Nazionale delle Ricerche, Rome, Italy, ${ }^{2}$ Department of Neuroscience, Imaging and Clinical Sciences, University 'Gabriele \\ d'Annunzio' of Chieti-Pescara, Chieti, Italy, ${ }^{3}$ Unit of Neurology, Neurophysiology, Neurobiology, Department of Medicine, \\ Università Campus Bio-Medico di Roma, Rome, Italy
}

Keywords: plasticity, synchrony, feedback, neurodynamics, recursive multiscale triadic principle

OPEN ACCESS

Edited by:

Plamen Ch. Ivanov,

Boston University, United States

Reviewed by:

Daniela Dentico,

University of Bari Aldo Moro, Italy

Yuan Yang,

University of Oklahoma, United States

*Correspondence:

Franca Tecchio

franca.tecchio@cnr.it

orcid.org/0000-0002-1325-5059

Specialty section:

This article was submitted to Fractal and Network Physiology,

a section of the journal

Frontiers in Physiology

Received: 24 September 2020 Accepted: 25 November 2020

Published: 15 December 2020

Citation:

Tecchio F, Bertoli M, Gianni E, L'Abbate T, Paulon L and Zappasodi F (2020) To Be Is To Become. Fractal

Neurodynamics of the Body-Brain Control System

Front. Physiol. 11:609768 doi: 10.3389/fphys.2020.609768
The Network Physiology field frames the multi-scale multi-dimensional nature of the body system emerging in the interaction among organs, which interplay via hemodynamic and metabolic functions under hormonal and neuronal controlling communication (Bashan et al., 2012; Ivanov and Bartsch, 2014; Bartsch et al., 2015; Ivanov et al., 2016; Lin et al., 2016). Thus, while the Network Physiology models networks consisting of organs (nodes) that are heterogeneous and connected by systems (connectors) of a still different nature, the brain is made up of elements that are at the same time nodes (soma of the neuron) and connectors (axons), so that the communicative-necessary and sufficient-nature confers to the sets of neurons the status of Network. Here we refer to Neuronal Networks $[\mathrm{NN}]$, which structurally include at least one node receiving inputs from the environment, and one node producing outputs to the environment; the $\mathrm{NN}$ connections are necessarily both negative and positive; every NN's node "necessarily" produces a pattern-OUT when the pattern-IN arrives, overall resulting in a specific local time course of the electrical neuronal activity, the local neurodynamics.

Here, grounding on existing knowledge, we propose a unique functional organizing principle-the feedback-synchrony-plasticity triad-which, governing the neuronal networks at multiple scales, emerges as a potential explanatory framework for the fractal properties exhibited by neurodynamics. In a translational perspective, via the strategy of "listening" to the body-brain organization by non-invasive electrophysiological techniques (electro- and magneto-encephalography and electromyography) integrated with "intervening" by non-invasive brain stimulation techniques, we exploited the communication means used by neuronal networks to enhance the capability of fighting symptoms secondary to neurodynamics dysfunctions. In other words, we introduce precision approaches to electroceuticals, i.e., the cure of ailments by means of electrical signals (Reardon, 2014).

\section{THE FEEDBACK-SYNCHRONY-PLASTICITY TRIADIC PRINCIPLE (FeeSyCy) GOVERNS THE BODY-BRAIN SYSTEM}

We consider the whole brain as a neurons' ensemble which coordinates the interaction of the body brain network with the environment, where input depends on the output and the other way round, the output depends on the input, working in a feedback loop. Via somatic, proprioceptive (Rossi et al., 1998; Fink et al., 2014), visual and auditory sensory receptors, our motor actions produce from the environment feedback, that our brain shapes dependently on the desired goal (Friston, 2018). This feedback loop stimulates our brain neurons inducing locally specific dynamic synchronizations among the nodes of dedicated functional networks (Tecchio et al., 2008; Gandolla et al., 2014). Such synchronizations 
within the network's subsystems imply a desynchronization of those very subsystems with the wider regions they are part of, resulting in a reduction of the resting-state high power of the cortical activity paced within the thalamocortical loops (Gent et al., 2018), e.g., alpha reactivity (Klimesch, 1999). In turn, these modulations of synchrony engage the system in adaptations either sustaining the execution as planned or enabling proper corrections (Fink et al., 2014). In this process, our neurons implement output changes following a key rule (Kandel and Schwartz, 1985): if two input signals reach the neuron together, the neuron increases its probability to fire (Hebb, 1949), that is to produce an action potential transmitting a message. Some authors indicate that the Hebbian rule subtends main trialand-error (Hoerzer et al., 2014) and imitation (Keysers and Gazzola, 2014) learning mechanisms. This continuous adaptation capability shapes the ability of our neurons to change their output according to what is required, quantified depending on the distance between the expected outcome and the current one. When the distance is small, behavioral adaptations emerge through the current network setup [working adaptation (Wolpert et al., 2011)]. When the distance is big, new skill acquisitions emerge through even huge structural changes (plastic adaptation, i.e., learning). A richness and complexity of molecular and cellular phenomena and of signaling, in continuous discovery, underlie the cellular and network modifications that implement the plastic adaptations. Plasticity mechanisms occurring at the synapses' level with non-unitary interplaying potentiation and depression phenomena (Malenka and Bear, 2004) are integrated by intrinsic plasticity mechanisms (Zhang and Linden, 2003) and changes in myelin multi-laminar sheaths that modulate the timing of information transmission between relay points through neural circuits, inducing changes in spike arrival-time, with which a high degree of precision controls the probability of activation (Gibson et al., 2014; Fields, 2015). It is supposed that Hebbain rules acting in day time, are supported during sleep spontaneous activity, by renormalizations of net synaptic strengths (Tononi and Cirelli, 2014) implementing homeostatic plasticity (Turrigiano and Nelson, 2004).

Notably, the feedback-synchrony-plasticity (FeeSyCy) triadic principle that governs motor control, controls the whole bodybrain system. We can recognize some paradigmatic examples of the breakup of one of the three links in the FeeSyCy chain, which generates the breakup of the whole process.

\section{Feedback Link Breakup}

The lack of auditory training and feedback condemned for centuries deaf individuals, despite owning intact motor executive functionality, to the inability to develop linguistic production, that is it condemned them to live as a deaf-mute (Sacks, 1989). The role of feedback is strongly proven by deaf people who grow nowadays. Starting from the last century, the teaching models and techniques -guided by neuroscientific comprehension-have definitely revolutionized the condition of deaf people, who now can, in parallel to the sign language, achieve an excellent production of language vocal expression by exploiting during their development the feedback about their produced words properly translated in signals from the spared sensory channels, mainly the visual one.

\section{Synchrony Link Breakup}

In dystonic individuals, despite proper sensory stimuli being transmitted via intact sensory systems, the impaired intracerebral synchronizations subtending the sensorimotor integration (Melgari et al., 2013), impairs the motor control (Abbruzzese and Berardelli, 2003).

\section{Plasticity Link Breakup}

Schizophrenic individuals are able to move and receive proper sensory feedback from the environment but cannot engage in proper adaptation due to neuronal inability to involve the metabolic chains and adapt the cells via plasticity (Ramocki and Zoghbi, 2008).

\section{THE FeeSyCy TRIADIC PRINCIPLE MANIFESTS ITSELF RECURSIVELY AT MULTIPLE SCALES}

\section{Single Neurons' Network}

In in-vitro primary cell culture of single cortical pyramidal neurons of postnatal rats, the synaptic changes implementing long-term potentiation and depression emerged as a function of incoming activity (Turrigiano et al., 1998; Sjöström and Nelson, 2002). Synaptic potentiation increases the postsynaptic firing rates in correlation with presynaptic activity, producing a positive feedback loop. Multiplicative scaling of synaptic strengths preserves relative differences between inputs, allowing a non-saturated implementation of Hebbian modifications (Hebb, 1949).

\section{Neuronal Pools' Network}

In functioning of multiple brain areas networks, a parallel capturing of bottom-up patterns of activation in sensorymotor areas occurs together with a top-down processing that selects sensory-motor activations to implement long-lasting storage. As memories organize themselves in central structures, they implement an active selection of sensory experience, proprioception and emotional knowledge for further learning (Barsalou, 1999).

\section{Body-Brain Network}

Deepening the paradigmatic example of motor execution, skilled actions require the actual gathering of sensory information, which is processed extracting what is relevant to the planned action. Such feedback comes from different types of information that the motor system uses as a learning signal, including error-based, reinforcement, observational and use-dependent information. In all cases, motor learning occurs implementing adaptations dependent on the distance between the expected and occurring inputs (Wolpert et al., 2011).

We can recognize an expression at the whole system level of the multi-scale recursive FeeSyCy principle in the human gait showing fractal dynamics (Hausdorff et al., 1996; Phinyomark et al., 2020) and also across species, in experimental data about 
food-searching strategies in insect, mammal and bird species (Edwards et al., 2007).

\section{WORKING AT MULTIPLE SCALES, THE FeeSyCy PRINCIPLE SUBTENDS A FRACTAL NEURODYNAMICS}

When a system presents the whole structure that is made up of single blocks, which are similar to the whole, and are in turn made of smaller blocks, similar to it and to the whole structure, it is a fractal. Its name comes from a non-integer number that quantifies its dimension. In our case, FD estimates on a time window the distance between the amplitudes of successive neuronal electrical activity points, in relationship with the time sampling.

Brain neurodynamics displays the so-called "power law" (He, 2011), i.e., the power of the signal generated by a neuronal population follows an exponential behavior. Among the multiple signals with a spectrum that distributes as power law, we propose the hypothesis that brain signals are fractal (Buzsaki and Mizuseki, 2014).

The findings from our laboratory support this hypothesis. We observed that the fractal dimension (FD) of EEG signals successfully senses the modulation of the brain activity in physiological conditions, related to aging (Zappasodi et al., 2015; Smits et al., 2016), circadian rhythm (Croce et al., 2018), behavioral states (Cottone et al., 2017) and neuronal networks' functional role (Marino et al., 2019), and the alterations of the brain activity in clinical conditions (Zappasodi et al., 2014; Smits et al., 2016; Porcaro et al., 2019). Notably, beyond being sensitive to the networks' state, FD offers a tool to parcel the cortex on the base of the local neurodynamics, complementing the Brodmann's cytoarchitectonics criterion (Cottone et al., 2017) (Figure 1).

\section{NEURONAL NETWORK SPOKEN LANGUAGE AND ELECTROCEUTICALS}

Nowadays the ability to develop therapeutic procedures by intervening on the body physiology by electric signals gives rise to the innovative branch in the medical field: the Electroceuticals (Reardon, 2014). Parallel to the need for technological advancements, they require further knowledge about the correct signals to be provided to the appropriate targets. We propose here a hypothesis on this matter, in the case of neuromodulation, the change of neuronal excitability.

By linking theoretical and experimental studies, the neuroscientific community is revealing network dynamics properties attuned with FeeSyCy mechanisms (Destexhe and Marder, 2004; Deco et al., 2011) that inspired our model of communication within neuronal networks. The model states that every $\mathrm{NN}$-were nodes can be made of neurons, groups of neurons or wider brain regions-develops a "language" shared by its nodes made of exchanged electric pattern, which dynamics' shape brings information (word, Neuronal Network Spoken Language). Notably, when assessing the fractal dimension of the bipolar EEG whole-brain signals we sensed phenomena sensed even by other measures. Noteworthy, when we assessed local neuronal ensemble neurodynamics, the fractal dimension, and not other measures, sensed in resting-state tiny changes with clinical relevance (Porcaro et al., 2019).

The neuroscientific community states that the efficacy of neuromodulation, the change of neuronal electric excitability, depends on the frequency of the stimulation in a regiondependent manner (Brinkman et al., 2016; Fusco et al., 2018), revealing that the intrinsic dynamics of the stimulation target enhances neuromodulation capability. In a seminal non-invasive transcranial electric stimulation (tES) study (Cottone et al., 2018), we proved that a current which mimics the endogenous dynamics

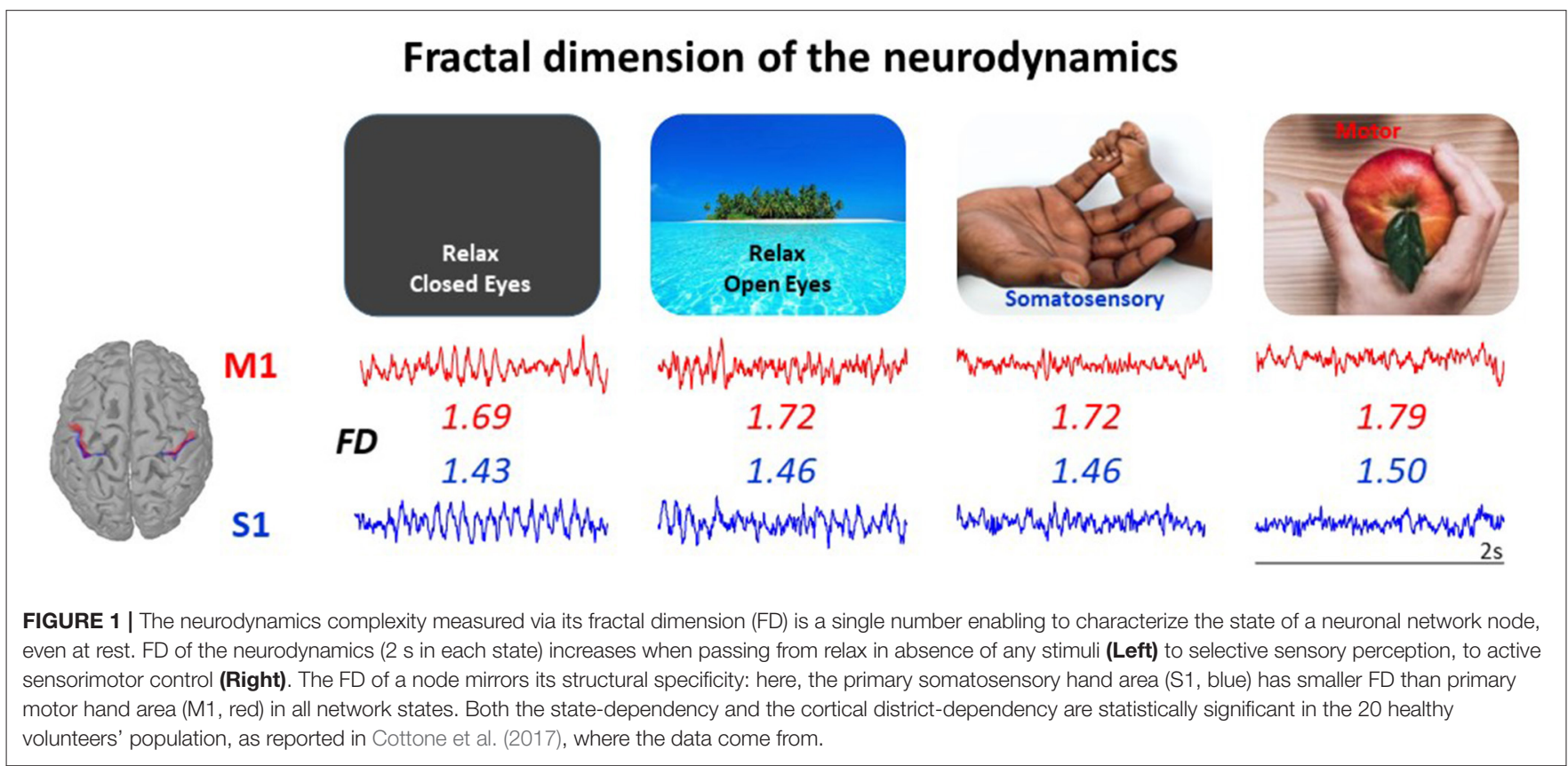


of the target neuronal pools, neuromodulates more efficiently than the sinusoid at a locally-tuned frequency, suggesting that structured patterns transmit entrainment more than a nonstructured stationary signal.

Near and more long-term future will see further electroceutical personalizations, by developing tools to "speak" the neuronal network language, thus better tuning the neuromodulation to the desired neuronal pool target and obtaining higher efficacy in compensating symptoms secondary to alterations of the neurodynamics, like depression, addiction, pain, fatigue.

This nature of the body-brain in continuous adaptive communication with the environment makes a continuously changing structure that is "to be is to become".

\section{REFERENCES}

Abbruzzese, G., and Berardelli, A. (2003). Sensorimotor integration in movement disorders. Mov. Disord. 51, 427-436. doi: 10.1002/mds.10327

Barsalou, L. W. (1999). Perceptual symbol systems. Behav. Brain Sci. 22, 577-609. doi: 10.1017/S0140525X99002149

Bartsch, R. P., Liu, K. K. L., Bashan, A., and Ivanov, P. C. (2015). Network physiology: how organ systems dynamically interact. PLOS ONE 10:e142143. doi: 10.1371/journal.pone.0142143

Bashan, A., Bartsch, R. P., Kantelhardt, J. W., Havlin, S., and Ivanov, P. C. (2012). Network physiology reveals relations between network topology and physiological function. Nat. Commun. 3:702. doi: 10.1038/ ncomms1705

Brinkman, L., Stolk, A., Marshall, T. R., Esterer, S., Sharp, P., Dijkerman, H. C., et al. (2016). Independent causal contributions of Alpha- and Betaband oscillations during movement selection. J. Neurosci. 36, 8726-8733. doi: 10.1523/JNEUROSCI.0868-16.2016

Buzsaki, G., and Mizuseki, K. (2014). The log-dynamic brain: how skewed distributions affect network operations. Nat. Rev. Neurosci. 15, 264-278. doi: $10.1038 / \mathrm{nrn} 3687$

Cottone, C., Cancelli, A., Pasqualetti, P., Porcaro, C., Salustri, C., and Tecchio, F. (2018). A new, high-efficacy, noninvasive transcranial electric stimulation tuned to local neurodynamics. J. Neurosci. 38, 586-594. doi: 10.1523/JNEUROSCI.2521-16.2017

Cottone, C., Porcaro, C., Cancelli, A., Olejarczyk, E., Salustri, C., and Tecchio, F. (2017). Neuronal electrical ongoing activity as a signature of cortical areas. Brain Struct. Funct. 222, 2115-2126. doi: 10.1007/s00429-016-1328-4

Croce, P., Quercia, A., Costa, S., and Zappasodi, F. (2018). Circadian rhythms in fractal features of EEG signals. Front. Physiol. 9:1567. doi: $10.3389 /$ fphys.2018.01567

Deco, G., Jirsa, V. K., and McIntosh, A. R. (2011). Emerging concepts for the dynamical organization of resting-state activity in the brain. Nat. Rev. Neurosci. 12, 43-56. doi: 10.1038/nrn2961

Destexhe, A., and Marder, E. (2004). Plasticity in single neuron and circuit computations. Nature 431, 789-795. doi: 10.1038/nature03011

Edwards, A. M., Phillips, R. A., Watkins, N. W., Freeman, M. P., Murphy, E. J., Afanasyev, V., et al. (2007). Revisiting Lévy flight search patterns of wandering albatrosses, bumblebees and deer. Nature 449, 1044-1048. doi: 10.1038/nature06199

Fields, R. D. (2015). A new mechanism of nervous system plasticity: activitydependent myelination. Nat. Rev. Neurosci. 16, 756-767. doi: 10.1038/nrn4023

Fink, A. J. P., Croce, K. R., Huang, Z. J., Abbott, L. F., Jessell, T. M., and Azim, E. (2014). Presynaptic inhibition of spinal sensory feedback ensures smooth movement. Nature 509, 43-48. doi: 10.1038/nature13276

Friston, K. (2018). Does predictive coding have a future? Nat. Neurosci. 21, 1019-1021. doi: 10.1038/s41593-018-0200-7

Fusco, G., Scandola, M., Feurra, M., Pavone, E. F., Rossi, S., and Aglioti, S. M. (2018). Midfrontal theta transcranial alternating current stimulation modulates

\section{AUTHOR CONTRIBUTIONS}

FT conceived the paper and supervised the writing. FT and FZ contributed to the writing of the original draft. MB contributed to figures creation. MB, TL, EG, and LP contributed to the writing and the editing of the manuscript. All authors reviewed and approved the final manuscript.

\section{ACKNOWLEDGMENTS}

The authors would like to thank Carlo Salustri very much for his careful sharing of our reasoning and tuning in communicating the contents of the Opinion.

behavioural adjustment after error execution. Eur. J. Neurosci. 48, 3159-3170. doi: 10.1111/ejn.14174

Gandolla, M., Ferrante, S., Molteni, F., Guanziroli, E., Frattini, T., Martegani, A., et al. (2014). Re-thinking the role of motor cortex: context-sensitive motor outputs? Neuroimage 91, 366-374. doi: 10.1016/j.neuroimage.2014.01.011

Gent, T. C., Bandarabadi, M., Herrera, C. G., and Adamantidis, A. R. (2018). Thalamic dual control of sleep and wakefulness. Nat. Neurosci. 21, 974-984. doi: 10.1038/s41593-018-0164-7

Gibson, E. M., Purger, D., Mount, C. W., Goldstein, A. K., Lin, G. L., Wood, L. S., et al. (2014). Neuronal activity promotes oligodendrogenesis and adaptive myelination in the mammalian brain. Science 344:1252304. doi: 10.1126/science.1252304

Hausdorff, J. M., Purdon, P. L., Peng, C. K., Ladin, Z., Wei, J. Y., and Goldberger, A. L. (1996). Fractal dynamics of human gait: stability of longrange correlations in stride interval fluctuations. J. Appl. Physiol. 80, 1448-1457. doi: 10.1152/jappl.1996.80.5.1448

He, B. J. (2011). Scale-free properties of the functional magnetic resonance imaging signal during rest and task. J. Neurosci. 31, 13786-13795. doi: 10.1523/JNEUROSCI.2111-11.2011

Hebb, D. O. (1949). Organization of Behavior. 1949th ed. New York, NY: John Wiley \& Sons, Ltd

Hoerzer, G. M., Legenstein, R., and Maass, W. (2014). Emergence of complex computational structures from chaotic neural networks through reward-modulated hebbian learning. Cereb. Cortex 24, 677-690. doi: $10.1093 /$ cercor/bhs348

Ivanov, P. C., and Bartsch, R. P. (2014). "Network physiology: mapping interactions between networks of physiologic networks," in Networks of Networks: The Last Frontier of Complexity. Understanding Complex Systems, eds G. D’Agostino, A. Scala (Cham: Springer). doi: 10.1007/978-3-319-03518-5_10

Ivanov, P. C. H., Liu, K. K. L., and Bartsch, R. P. (2016). Focus on the emerging new fields of network physiology and network medicine. New J. Phys. 18:100201. doi: 10.1088/1367-2630/18/10/100201

Kandel, E., and Schwartz, J. (1985). Principles of Neural Sciences. 2nd Edn. New York, NY, Oxford, Amsterdam: Elsevier.

Keysers, C., and Gazzola, V. (2014). Hebbian learning and predictive mirror neurons for actions, sensations and emotions. Philos. Trans. R. Soc. B Biol. Sci. 369:20130175. doi: 10.1098/rstb.2013.0175

Klimesch, W. (1999). EEG alpha and theta oscillations reflect cognitive and memory performance: a review and analysis. Brain Res. Rev. 29, 169-195. doi: 10.1016/S0165-0173(98)00056-3

Lin, A., Liu, K. K. L., Bartsch, R. P., and Ivanov, P. C. (2016). Delay-correlation landscape reveals characteristic time delays of brain rhythms and heart interactions. Philos. Trans. R. Soc. A Math. Phys. Eng. Sci. 374:20150182. doi: 10.1098/rsta.2015.0182

Malenka, R. C., and Bear, M. F. (2004). LTP and LTD: an embarrassment of riches. Neuron 44, 5-21. doi: 10.1016/j.neuron.2004.09.012

Marino, M., Liu, Q., Samogin, J., Tecchio, F., Cottone, C., Mantini, D., et al. (2019). Neuronal dynamics enable the functional differentiation of resting 
state networks in the human brain. Hum. Brain Mapp. 40, 1445-1457. doi: $10.1002 / \mathrm{hbm} .24458$

Melgari, J. M., Zappasodi, F., Porcaro, C., Tomasevic, L., Cassetta, E., Rossini, P. M., et al. (2013). Movement-induced uncoupling of primary sensory and motor areas in focal task-specific hand dystonia. Neuroscience 250, 434-445. doi: 10.1016/j.neuroscience.2013.07.027

Phinyomark, A., Larracy, R., and Scheme, E. (2020). Fractal Analysis of Human Gait Variability via Stride Interval Time Series. Front. Physiol. 11:333. doi: $10.3389 /$ fphys.2020.00333

Porcaro, C., Cottone, C., Cancelli, A., Rossini, P. M., Zito, G., and Tecchio, F. (2019). Cortical neurodynamics changes mediate the efficacy of a personalized neuromodulation against multiple sclerosis fatigue. Sci. Rep. 9:18213. doi: 10.1038/s41598-019-54595-Z

Ramocki, M. B., and Zoghbi, H. Y. (2008). Failure of neuronal homeostasis results in common neuropsychiatric phenotypes. Nature 455, 912-918. doi: $10.1038 /$ nature 07457

Reardon, S. (2014). Electroceuticals spark interest. Nature 511:18. doi: $10.1038 / 511018$ a

Rossi, S., Pasqualetti, P., Tecchio, F., Sabato, A., and Rossini, P. M. (1998). Modulation of corticospinal output to human hand muscles following deprivation of sensory feedback. Neuroimage 8, 163-175. doi: 10.1006/nimg.1998.0352

Sacks, O. (1989). Seeing Voices: A Journey into the World of the Deaf. 1989th Edn., ed. California, CA: University of California Press Berkeley.

Sjöström, P. J., and Nelson, S. B. (2002). Spike timing, calcium signals and synaptic plasticity. Curr. Opin. Neurobiol. 12, 305-314. doi: 10.1016/S0959-4388(02)00325-2

Smits, F. M., Porcaro, C., Cottone, C., Cancelli, A., Rossini, P. M., and Tecchio, F. (2016). Electroencephalographic fractal dimension in healthy ageing and alzheimer's disease. PLoS ONE 11:e0149587. doi: 10.1371/journal.pone. 0149587

Tecchio, F., Zappasodi, F., Porcaro, C., Barbati, G., Assenza, G., Salustri, C., et al. (2008). High-gamma band activity of primary hand cortical areas: a sensorimotor feedback efficiency index. Neuroimage 40, 256-264. doi: 10.1016/j.neuroimage.2007.11.038

Tononi, G., and Cirelli, C. (2014). Sleep and the price of plasticity: from synaptic and cellular homeostasis to memory consolidation and integration. Neuron 81 , 12-34. doi: 10.1016/j.neuron.2013.12.025

Turrigiano, G. G., Leslie, K. R., Desai, N. S., Rutherford, L. C., and Nelson, S. B. (1998). Activity-dependent scaling of quantal amplitude in neocortical neurons. Nature 391, 892-896. doi: 10.1038/36103

Turrigiano, G. G., and Nelson, S. B. (2004). Homeostatic plasticity in the developing nervous system. Nat. Rev. Neurosci. 5, 97-107. doi: 10.1038/nrn1327

Wolpert, D. M., Diedrichsen, J., and Flanagan, J. R. (2011). Principles of sensorimotor learning. Nat. Rev. Neurosci. 12, 739-751. doi: 10.1038/nrn3112

Zappasodi, F., Marzetti, L., Olejarczyk, E., Tecchio, F., and Pizzella, V. (2015). Age-related changes in electroencephalographic signal complexity. PLoS ONE 10:e0141995. doi: 10.1371/journal.pone.0141995

Zappasodi, F., Olejarczyk, E., Marzetti, L., Assenza, G., Pizzella, V., and Tecchio, F. (2014). Fractal dimension of EEG activity senses neuronal impairment in acute stroke. PLoS ONE 9:e100199. doi: 10.1371/journal.pone.0100199

Zhang, W., and Linden, D. J. (2003). The other side of the engram: experiencedriven changes in neuronal intrinsic excitability. Nat. Rev. Neurosci. 4, 885-900. doi: $10.1038 / \mathrm{nrn} 1248$

Conflict of Interest: The authors declare that the research was conducted in the absence of any commercial or financial relationships that could be construed as a potential conflict of interest.

Copyright (C) 2020 Tecchio, Bertoli, Gianni, L'Abbate, Paulon and Zappasodi. This is an open-access article distributed under the terms of the Creative Commons Attribution License (CC BY). The use, distribution or reproduction in other forums is permitted, provided the original author(s) and the copyright owner(s) are credited and that the original publication in this journal is cited, in accordance with accepted academic practice. No use, distribution or reproduction is permitted which does not comply with these terms. 